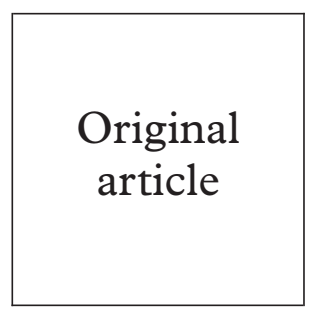

\title{
A longitudinal study of HPV detection and cervical pathology in HIV infected women
}

\author{
H A Cubie, A L Seagar, G J Beattie, S Monaghan, A R W Williams
}

Objective: To monitor the presence and persistence of high risk (HR) human papillomavirus (HPV) in cervical brushings from HIV infected women.

Methods: Prospective observational cohort study of HIV infected women. Women were enrolled from the cohort of 164 HIV infected women who attend the colposcopy clinic at the Edinburgh Regional Infectious Diseases Unit. A single cervical brush scrape was obtained from 39 women and two or more samples from 63 women who attended regularly at approximately 6 monthly intervals. HPV typing was carried out using a commercial hybrid capture assay (HCA). Details of antiretroviral therapy, cytological assessment, and histological evaluation were made available and the interrelation with HR-HPV detection analysed.

Results: Abnormal cervical cytology, particularly of low grade, was common in these HIV infected women. HR-HPV types were detected in $25 \%$ of the women with normal cytology, while over $80 \%$ of those with abnormal cytology of any grade were HR-HPV positive. Persistent HR-HPV, as defined by two or more consecutive HPV positive results, was common and found in 27/63 women from whom multiple samples were obtained. HR-HPV was detected at high levels whether or not patients were receiving antiretroviral therapy. Profound immunosuppression was not necessarily associated with progression of cervical disease and no cases of invasive cervical disease were seen.

Conclusion: While mild dyskaryosis (low grade squamous intraepithelial lesion (LSIL)) and persistence of HR-HPV are common in HIV infected women in Edinburgh, regular cytological and colposcopic evaluation with appropriate intervention and treatment appears to limit the progression of cervical disease.

(Sex Transm Inf 2000;76:257-261)

Keywords: human papillomavirus; HIV; cervical disease

\section{Introduction}

Both men and women infected with HIV show an increased frequency of anogenital human papillomavirus (HPV) infection. ${ }^{1-4}$ The severity of HPV associated cervical disease has been linked to increasing immunological impairment. $^{5-7}$ HIV infected women with reduced CD4 lymphocyte counts appear to carry cervical HPV more frequently and more persistently than HIV women whose CD4 counts are not reduced. ${ }^{8}$ However, increased cervical disease is not always found with increasing immunosuppression in HIV infected women. ${ }^{9}$ Norman and McCarthy point out the major difficulties in adequate matching of HIV seropositive and negative study groups and the wide variation in measures used to assess cervical abnormality and in 1994 suggested that many of the difficulties could be circumvented by longitudinal studies over at least a decade.

In the Edinburgh cohort of HIV infected women a clear association between abnormal cervical cytology and HIV infection has been shown, ${ }^{10}$ with $35 \%$ of the women having some degree of histologically proved cervical intraepithelial neoplasia (CIN). ${ }^{11}$ In addition, $34 \%$ of those with CIN had recurrences associated with reduced CD4 counts within 2 years of treatment. ${ }^{11}$ Recurrent CIN in HIV positive women has also been noted by others. ${ }^{12-13}$ Progression of cervical disease is most commonly associated with high risk (HR) human papillomavirus (HPV) types, especially
HPV 16, 18, 31, and 45, which have been found to be present in more than $90 \%$ of cervical carcinomas worldwide. ${ }^{14}$

While an increase in anal intraepithelial neoplasia and carcinoma in HIV infected men has been reported in the United States ${ }^{1}$ and in Edinburgh (A McMillan, personal communication), such an increase is not always observed. ${ }^{4}$ Similarly, an increase in cervical carcinoma has been noted in women in the United States, ${ }^{15}$ but there is no documented evidence of an increase in cervical carcinoma in HIV infected women in Scotland.

The Edinburgh group of HIV infected women was one of the first well documented groups where infection arose largely as a result of injecting drug use and needle sharing. The majority seroconverted over a fairly short period of time in the mid-1980s and therefore provide a good model for the study of the natural history of HIV associated diseases. The stable well defined nature of this group together with their regular attendance at clinics makes it an ideal population for a prospective study. We report here the cumulative results covering a period of 3 years in which we have studied HPV presence and cervical disease patterns in relation to clinical management.

\section{Patients and methods}

STUDY GROUP AND SAMPLE COLLECTION The cohort comprises 164 HIV infected women who have ever attended the colposcopy clinic at the Regional Infectious Diseases Unit
Accepted for publication 20 March 2000 
in Edinburgh. To date, 106 women have been enrolled in the study. The age range at enrolment was 23-55 years, with a mean of 33 years and they are representative of the homogeneous population resident in south east Scotland. Women are invited to attend at 6 monthly intervals for routine review, including cervical cytology and colposcopic examination, with cervical punch biopsies where appropriate.

At each attendance, a sample of cervical brushings was obtained by Cervex brush and collected into preservative fluid. The sample was divided into equal aliquots, centrifuged to deposit cells, and thoroughly resuspended in transport medium (STM; Digene Diagnostics, Silver Spring, MD, USA) for HPV hybrid capture testing (Digene Diagnostics). To date, 298 samples have been received from the cohort. The analysis presented here is based on 226 samples from the 63 women from whom we have already received two or more samples. It excludes 39 women from whom only one sample has been received and a further 33 for whom cytology information is not available or the sample is inadequate. Collection of samples is continuing and further analysis will be carried out after 5 years of follow up. Ethical permission for this study was obtained from Lothian Health. Patients with histological evidence of CIN of any grade were treated using either cold coagulation or, more recently, loop diathermy in the conventional manner.

CD4 counts, cytology, and histology were carried out using standard diagnostic procedures and results made available for this study. Cytology definitions of borderline, mild, and moderate/severe dyskaryosis follow the UK convention and are equivalent to ASCUS (but including some HPV infection), LSIL, and HSIL respectively in the Bethesda classification. Histologically, low grade cervical disease is reported as koilocytosis $(\mathrm{K}+) / \mathrm{CIN}$ I and high grade disease as CIN II/III. HIV RNA viral load results (copies/ml) obtained diagnostically by the Roche Amplicor system were also made available.

HPV DETECTION

HPV hybrid capture assay (HCA) is a sandwich capture hybridisation assay for qualitative detection of HR-HPV types, using a specific HPV RNA probe cocktail and an amplified chemiluminescence detection system. The emission of light is measured as relative light units (RLUs) and is proportional to the amount of target DNA present. Samples with a RLU/cut off value $>1$ indicate a positive result for any of the HR-HPV types present in the probe pool. First generation HCA (HCA I), a tube based assay detecting nine HR-HPV types, was used at the beginning of this study and was replaced by the second generation test (HCA II) as soon as it became available. This assay is performed using microtitre plates, has greater sensitivity, and detects more HPV types. Wherever possible, testing of early samples has been repeated using HCA II with the high risk probe pool for $13 \mathrm{HPV}$ types-16, 18, $31,33,35,39,45,51,52,56,58,59$, and 68 .

\section{Results}

CYTOLOGICAL AND HISTOLOGICAL RESULTS

Cervical smears in HIV positive patients were assessed under standard diagnostic conditions. Where histology was available, there was less correlation than expected between the cytology and histology. Review of high grade smears with a CIN I or negative histology result was carried out by the same cytopathologist (ARWW). This led to reclassification of three cases of moderate to mild dyskaryosis and one confirmed case of severe dyskaryosis as CIN II. The original and review data are presented in figure 1 . In the cohort of $164 \mathrm{HIV}$ infected women, analysis of histological abnormalities showed that high grade CIN (CIN II/III) reached a peak in 1990-2 (fig 2) and then dropped sharply. Low grade CIN (CIN I) increased between 1987-97 but now also appears to be decreasing.

DETECTION OF HIGH RISK HPV TYPES

Early samples were tested with HCA I and retested by HCA II when the sample was still available. The results with first and second generation assays were compared. In this small

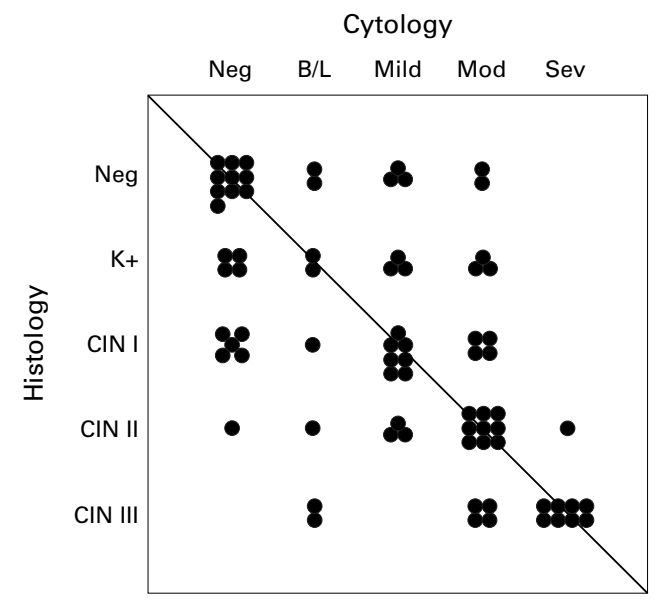

Figure 1 Scattergram showing correlation between cytological and histological diagnoses in $39 \mathrm{HIV}$ seropositive women from whom matching specimens were obtained.

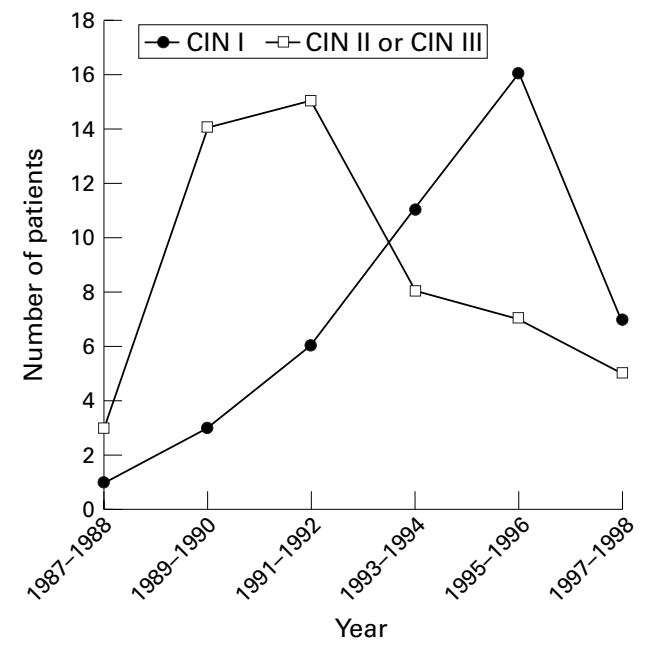

Figure 2 Occurrence of cervical intraepithelial neoplasia (CIN) in HIV infected women attending the Regional Infectious Diseases Unit, Edinburgh over a 12 year period. 
group of samples, concordance was shown in 77/90 (85.6\%). Unlike HCA I, a clear distinction between positive and negative was observed with HCA II, with no equivocal results being obtained.

The degree of abnormality observed cytologically together with the percentage of HR-HPV positive samples found was comparable whether first, last, or all samples were considered. Twenty three per cent of smears with normal cytology were HR-HPV positive; this increased to $70 \%$ overall in those with borderline smears, $91 \%$ in those with mild dyskaryosis, and $95 \%$ in those with moderate/ severe dyskaryosis (table 1). The highest RLU indices were generally obtained in samples with a significant degree of squamous dyskaryosis. In contrast, $85 \%$ of results with negative or equivocal RLU indices came from samples with normal cytology (table 2).

RELATION BETWEEN HIV SEROPOSITIVITY, HPV DETECTION, AND IMMUNOSUPPRESSION

At high HIV RNA viral loads (>50 000 copies $\times 10^{6} / 1$ ), the percentage of HR-HPV positive smears associated with a borderline cytology result was increased (table 3 ). With HIV viral load below 500 copies $\times 10^{6} / 1$, the percentages of HR-HPV positive smears were similar to percentages found in an HIV seronegative group (data not included; see Discussion). Four women with high HIV viral load and HPV positive smears had negative cytology both at initial assessment and on review.

Investigation of the interaction between HIV viral load and treatment, HR-HPV, cytological and histological abnormalities, and falling CD4 counts was confined to women from whom multiple samples were obtained. Forty eight women provided three or more samples, with six or more samples being obtained from 12 women. Individual patient profiles show considerable variation and representative examples are shown in figure 3. For example, P1 has a fluctuating CD 4 count, high HIV viral load despite antiretroviral therapy, persistent HPV, and continuing high grade cervical disease. In contrast, P3 has a low CD4

Table 1 HR-HPV DNA detection in samples of cervical brushings from women infected with HIV

\begin{tabular}{llll}
\hline & First sample (\%) & Last sample (\%) & All samples (\%) \\
\hline Unsatisfactory & $2 / 5(40)$ & $1 / 2$ & $3 / 8(38)$ \\
Normal & $9 / 36(25)$ & $11 / 44(25)$ & $33 / 141(23)$ \\
Borderline (ASCUS) & $7 / 12(58)$ & $4 / 7(57)$ & $23 / 33(70)$ \\
Mild dyskaryosis & $6 / 6(100)$ & $6 / 7(86)$ & $20 / 22(91)$ \\
Moderate and severe dyskaryosis & $3 / 4(75)$ & $3 / 3(100)$ & $21 / 22(95)$ \\
Total & $\mathbf{2 7 / 6 3 ( 4 3 )}$ & $\mathbf{2 5 / 6 3 ( 4 0 )}$ & $\mathbf{1 0 0 / 2 2 6 ( 4 4 )}$ \\
\hline
\end{tabular}

Table 2 Relation between degree of HR-HPV positivity and increasing cervical cytology

\begin{tabular}{llrlllr}
\hline \multirow{5}{*}{$\begin{array}{l}\text { HCA II result } \\
\text { (as RLU index) }\end{array}$} & U/S & Normal & Borderline & $\begin{array}{l}\text { Mild } \\
\text { dyskaryosis }\end{array}$ & $\begin{array}{l}\text { Mod/severe } \\
\text { dyskaryosis }\end{array}$ & Total \\
\cline { 2 - 6 } & 5 & 108 & 10 & 2 & 1 & 126 \\
\hline Negative & 0 & 1 & 0 & 0 & 0 & 1 \\
Equivocal & 3 & 17 & 13 & 6 & 4 & 43 \\
Positive (RLUI <10) & 0 & 13 & 5 & 4 & 3 & 25 \\
Positive (RLUI <100) & 0 & 2 & 5 & 9 & 12 & 3 \\
Positive (RLUI <1000) & 0 & 0 & 0 & 1 & 2 & $\mathbf{2 2 6}$ \\
Positive (RLUI >1000) & 0 & $\mathbf{1 4 0}$ & $\mathbf{3 3}$ & $\mathbf{2 1}$ & $\mathbf{2 2}$ & \\
Total & $\mathbf{8}$ & & & & & \\
\hline
\end{tabular}

Table 3 Relation between HIV viral load, detection of HR-HPV, and cytology

\begin{tabular}{lllrl}
\hline $\begin{array}{l}\text { Viral load } \\
\left(\text { copies } \times 10^{6} / l\right)\end{array}$ & $\begin{array}{l}\text { Smear } \\
\text { total }\end{array}$ & Cytology & Number & $\begin{array}{l}\text { HPV } \\
\text { positivity (\%) }\end{array}$ \\
\hline$<500$ & 32 & Neg & 24 & $7(29)$ \\
& & B/L & 4 & $1(25)$ \\
& & Mild & 3 & $2(67)$ \\
$500-5000$ & \multirow{2}{*}{26} & Mod/sev & 1 & $1(100)$ \\
& & Neg & 20 & $6(30)$ \\
& & B/L & 4 & $3(75)$ \\
& & Mild & 1 & 0 \\
$5000-50000$ & 37 & Ned & 23 & $1(100)$ \\
& & B/L & 6 & $4(17)$ \\
& & Mild & 3 & $3(100)$ \\
$>50000$ & 34 & Mod/sev & 5 & $5(100)$ \\
& & Neg & 16 & $4(25)$ \\
& & B/L & 6 & $6(100)$ \\
& & Mild & 5 & $5(100)$ \\
& & Mod/sev & 7 & $7(100)$ \\
\hline
\end{tabular}

count $\left(<100\right.$ cells $\left.\times 10^{6} / 1\right)$, HIV viral load currently controlled with triple therapy, but has persistent HPV and some cervical disease. $\mathrm{P} 4$ has a reasonable CD4 count (140-831 cells $\left.\times 10^{6} / 1\right)$, despite an increasing HIV viral load following discontinuation of triple therapy, has persistent HR-HPV, but no significant cervical disease. The progression of cervical disease in the presence of severe immunosuppression and HPV persistence was a rare event in this group, with only three examples being observed since the commencement of the study. In 27/63 women, persistent HR-HPV infection was noted, as defined by two or more consecutive HR-HPV positive results. In addition, HR-HPV has been detected in every sample taken since enrolment from 16 of these women. No microinvasive or invasive cervical cancers have been detected.

\section{Discussion}

In this HIV infected cohort, approximately $23 \%$ samples from women with cytologically normal smears were found to harbour HRHPV, with $70 \%$ of borderline and over $90 \%$ of dyskaryotic smears also HR-HPV positive. Using HCA II, we have detected a similar percentage of HR-HPV positive samples (25\%) in a group of uninfected women with normal cytology, while the percentage of positives in uninfected women with cytological abnormalities was lower (48\%; data not included). This agrees with a study from New York ${ }^{5}$ which showed a higher prevalence of HPV in HIV seropositive women, while a similar HPV prevalence in both HIV positive and negative women was reported recently in an Italian study. ${ }^{16}$

The principal test used in this study was a second generation HCA, which in our hands and in other studies has been shown to have comparable sensitivity to PCR. Clavel et $a l^{17}$ recently suggested that viral load estimated by HCA was not a reliable indicator of high grade lesions. We analysed the RLU indices for all samples (table 2) and found that $75 \%$ of samples with RLU indices between 100-1000 and $100 \%$ of those with RLU indices over 1000 showed cytological abnormalities, compared with $10 \%$ of those with RLU indices less than 100 , suggesting some correlation of increasing viral load with increasing cytological abnor- 
P1

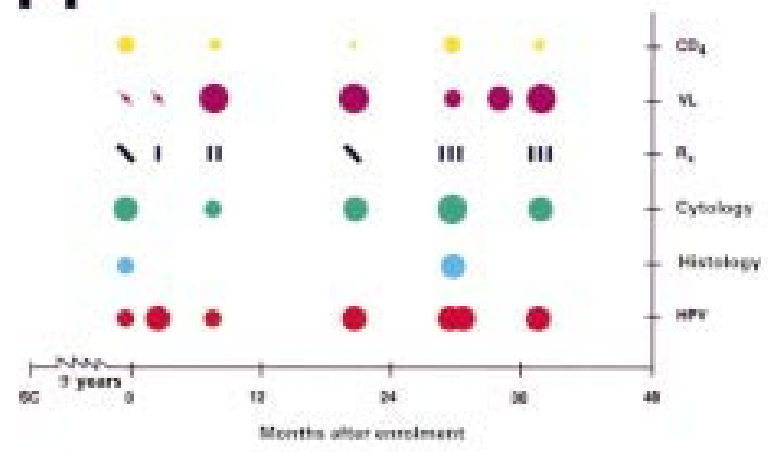

P3

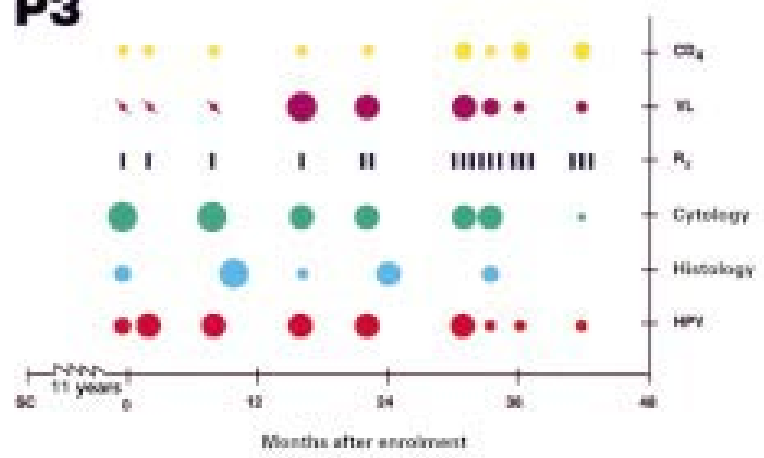

P5

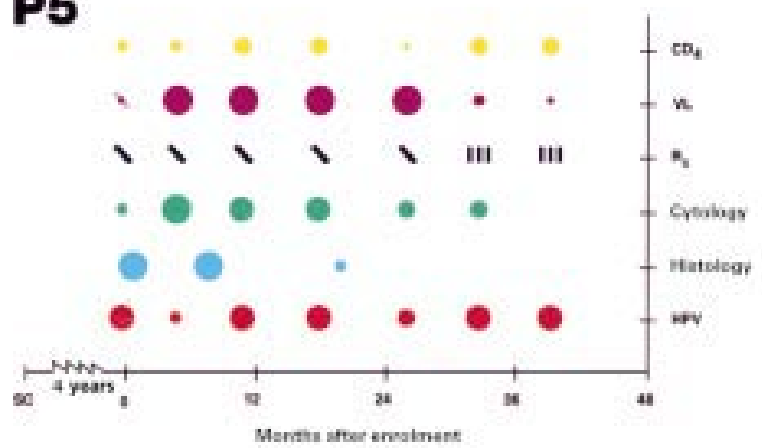

P2

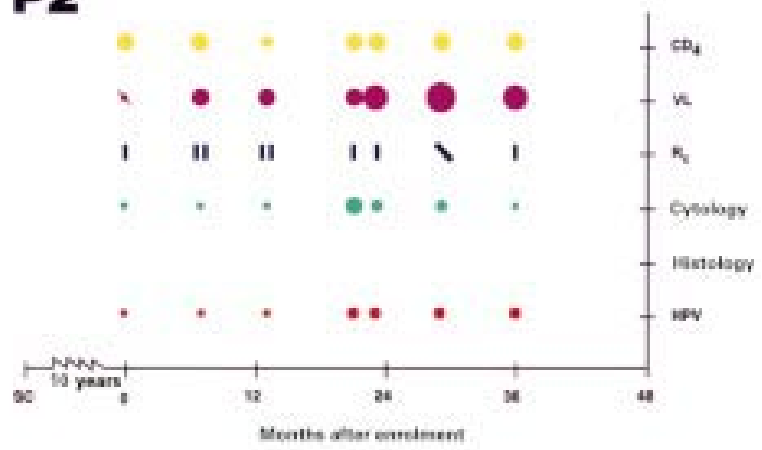

P4

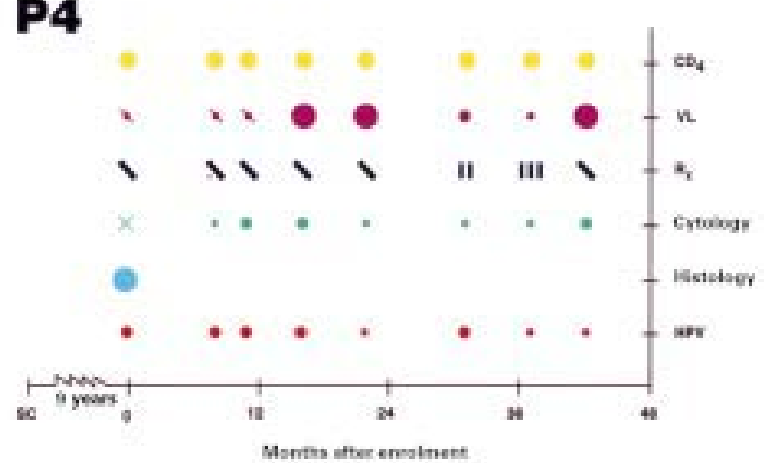

Figure 3 Individual patient profiles (P1, P2, P3, P4, and P5), showing variation in HIV viral load, HPV status, cervical cytology, CD4 counts, and antiretroviral therapy over time since sample collection began.

mality using HCA. However, a recent paper by Swan $e t a l^{18}$ concluded that the clinical usefulness of HPV quantification required reassessment and was assay dependent.

The high prevalence of some degree of dyskaryosis and/or CIN in many HIV infected women confirms our previous findings, ${ }^{10} 11$ while the relative lack of more severe cervical disease warrants further study. Indeed, the progression of cervical disease in the presence of increasing immunosuppression and HPV persistence was a rare event in this group with no microinvasive or invasive cervical cancers being detected. Invasive cervical cancer was included in the expanded definition of AIDS case definition in $1993^{19}$ and in the same year in Scotland, two cases were identified in which invasive cervical cancer was the initial AIDS defining illness. ${ }^{20}$ No further cases havebeen reported however. Similar observations have been made by other groups, ${ }^{12}$ questioning the relevance of including cervical cancer as an AIDS defining illness in HIV infected women.
It is interesting to speculate that the development of dyskaryosis and CIN in HIV positive women may simply be a marker of widespread immunosuppression rather than indicating likely progression to malignancy. Nevertheless, the development of cytological and histological abnormalities in HIV infected women appears to be complex and the impact of prolonged and at times profound immunosuppression may bring into play factors and interactions not seen elsewhere. While HIV has been suggested as an independent factor in the development of cervical disease, ${ }^{21}$ a recent study from Palefsky's group ${ }^{22}$ suggests that HIV positive women with a CD4 count of $<200$ cells $\times 10^{6} / 1$ were at highest risk of HPV infection regardless of HIV viral load. Furthermore, both enhancing and competitive interactions between HIV and viruses such as cytomegalovirus have been reported in vitro. ${ }^{23}$ Similarly, cellular changes induced by HIV could alter the molecular regulation of HPV. The impact of therapeutic interventions, 
antiretroviral therapy, and altered lifestyle on the development of CIN is difficult to assess. However a recent French study ${ }^{24}$ suggests that active antiretroviral therapy can result in a reduced prevalence of squamous intraepithelial neoplasia (SIL) despite persistent HR-HPV infection. A reduction in prevalence from $69 \%$ to $53 \%$ over a 5 month study period was observed and included regression from high grade to low grade lesion and from low grade to normal. This is an extremely important observation which requires further study in a larger group over a longer period.

Recommendations have been made for routine and regular colposcopy in this population as a means of screening for cervicovaginal disease ${ }^{25}$ and in the United Kingdom the need to develop national guidelines regarding the frequency of cervical cytology has already been identified. ${ }^{26}$ The Edinburgh cohort of HIV infected women is a well defined group who continue to provide a unique opportunity to study the interactions between HIV, HPV, and the development of cervical disease.

We are grateful to the Royal Infirmary of Edinburgh Cancer Fund for support for this project and to Digene Corporation for provision of HCA II kits to allow this study to continue. We are also grateful to staff in the Regional Clinical Virology Laboratory for access to HIV viral load data and to Dr A McMillan for critical review of the manuscript.

Contributors: HAC initiated and oversaw the project, including making grant applications and drafting the manuscript; ALS carried out the HPV testing and did most of the data collation and analysis; GJB was responsible for the clinical management and review of all the patients; SM retrieved relevant diagnostic
results from case notes and contributed to data analysis; ARWW carried out all histological and abnormal cytology assessments on all specimens from the patients and reviewed all available cases of discrepant results.

1 Palefsky JM. Anal human papillomavirus infection and anal cancer in HIV-positive individuals:an emerging problem. AIDS 1992;8:283-95.

2 Wright TC, Ellerbrock TV, Chiasson MA, et al. Cervical intraepithelial neoplasia in women infected with human immunodeficiency virus:prevalence, risk factors and validity of Papanicolaou smears. Obstet Gynecol 1994;84:591-7.

3 Six C, Heard I, Bergeron C, et al. Comparative prevalence,incidence and short-term prognosis of cervical squamous intraepithelial lesions amongst HIV-positive and negative women. AIDS 1998;12:1047-56.

4 Lacey HB, Wilson GE, Tilston P, et al. A study of anal intraepithelial neoplasia in HIV positive homosexual men. Sex Transm Inf 1999;75:172-7.

5 Sun XW, Kuhn L, Ellerbrock T, et al. Human papillomaviSun XW, Kuhn L, Ellerbrock T, et al. Human papillomavirus infection in women infected with the human

6 Johnson JC, Burnett AF, Willet GD, et al. High frequency of latent and clinical human papillomavirus cervical infections in immunocompromised human immunodeficiency virus-infected women. Obstet Gynecol 1992;79:321-7.
7 Maiman M, Fruchter RS, Guy L, et al. Human immunodeficiency virus infection and invasive cervical carcinoma. deficiency virus infection

8 Vernon SD, Reeves WC, Clancy KA, et al. A longitudinal study of human papillomavirus DNA detection in human immunodeficiency virus type-1 seropositive and seronegative women. F Infect Dis 1994;169:1108-12.

9 Norman SG, McCarthy KH. Gynaecological disease in women infected with the human immunodeficiency virus. Contemp Rev Obstet Gynecol 1994;6:46-50.

10 Johnstone FD, McGoogan E, Smart GE, et al. A population-based, controlled study of the relation between HIV infection and cervical neoplasia. Br f Obstet Gynaecol 1994;101:986-91.

11 Beattie GJ, Williams ARW, Farquarson DIM, et al. The incidence and recurrence of CIN in HIV positive women. incidence and recurrence of CIN in HIV

12 Fruchter RG, Maiman M, Sedlis A, et al. Multiple recurrences of cervical intraepithelial neoplasia in women with the human immunodeficiency virus. Obstet Gynecol 1996;87:338-44.

13 Adachi A, Fleming I, Burk RD, et al. Women with human immunodeficiency virus infection and abnormal Papanicolaou smears: a prospective study of colposcopy and clinical outcome. Obstet Gynecol 1993;81:372-7.

14 Bosch FX, Manos MM, Munoz N, et al. Prevalence of human papillomavirus in cervical cancer:a worldwide perhuman papillomavirus in cervical cancer: a worldwide per(IBSCC) Study Group. 7 Natl Cancer Inst 1995;87:796802.

15 Klevens RM, Fleming PL, Mays MA, et al. Characteristics of women with AIDS and invasive cervical cancer. Obstet Gynecol 1996;88:169-273.

16 Cappiello G, Garbuglia AR, Salvi R, et al. HIV infection increases the risk of squamous intra-epithelial lesions in women with HPV infection:an analysis of HPV genotypes. Int $\mathcal{F}$ Cancer 1997;72:982-6.

17 Clavel C, Masure M, Putaud I, et al. Hybrid Capture II, a new sensitive test for human papillomavirus detection. Comparison with hybrid capture I and PCR results in cervical lesions. F Clin Pathol 1998;51:737-40.

18 Swan DC, Tucker RA, Tortolero-Luna G, et al. Human papillomavirus (HPV) DNA copy number is dependent on grade of cervical disease and HPV type. 7 Clin Microbiol 1999;37:1030-4.

9 Centers for Disease Control and Prevention. 1993 Revised classification system for HIV infection and expanded surveillance case definition for AIDS among adolescents and adults. $M M W R$ 1992;41:1-19.

20 The Scottish Centre for Infection and Environmental Health. HIV and AIDS surveillance in Scotland 1991-1998. Edinburgh: Scottish Centre for Infection and Environmental Health, 1999:18.

21 Ho GYF, Burk RD, Fleming I, et al. Risk of genital human papillomavirus infection in women with human immunopapillomavirus infection in women with human immuno1994;56:788-92.

22 Palefsky JM, Minkoff H, Kalish LA, et al. Cervicovaginal human papillomavirus infection in human immunodeficiency virus-1 (HIV)-positive and high-risk HIVnegative women. F Natl Cancer Inst 1999;91:226-35.

23 Koval V, Jault FM, Pal PG, et al. Differential effects of human cytomegalovirus on integrated and unintegrated human immunodeficiency virus sequences. 7 Virol 1995; 69:1645-51.

24 Heard I, Schmitz V, Costagliola D, et al. Early regression of cervical lesions in HIV-seropositive women receiving highly active antiretroviral therapy. AIDS 1998;12:1459-64.

25 Maiman M, Tarricone N, Viera J, et al. Colposcopic evaluation of human immunodeficiency virus seropositive women. Obstet Gynecol 1991;78:84-8.

26 Jungmann EMA, Smith NA, Bradbeer C, et al. An audit of cervical cytological screening amongst HIV-positive women. Int F STD AIDS 1998;9:301-2. 